\title{
What Can Observations of Young Binaries Tell Us about Binary Formation?
}

\author{
Cathie J. Clarke \\ Institute of Astronomy, Madingley Road, Cambridge CB3 OHA, \\ England.
}

\begin{abstract}
In this paper I discuss three areas in which observational data is likely to have a strong impact on theoretical ideas about binary star formation: the collation of binary statistics for different primary masses, the acquisition of spectral energy distributions for individual components within pre-main sequence binaries and the use of binary statistics to constrain the mix of star forming regions that must combine to form the field. Theoretical and observational work is ongoing in each of these areas. It is suggested that for observers an important future task is to remeasure the wide binary population among local field stars, whilst for theorists a problem yet to be addressed is how the mass of a molecular cloud core affects its binary fragmentation properties.
\end{abstract}

\section{Introduction}

The growing dataset of high quality observational data on binary pairing properties offers the opportunity, for the first time, to place constraints on viable mechanisms for binary star formation. This situation contrasts strongly with that of a few years ago, when theoretical models only had to demonstrate feasibility, as opposed to the ability to reproduce a specific set of observational parameters. Of particular significance is the recent acquisition of binary statistics for volume limited samples of stars of different primary masses, since, as will be shown below, this provides a discriminant between contending models. It should be noted that the measurement of a companion mass function for primaries of a single mass - as in the landmark survey of Duquennoy and Mayor 1991 - provides considerably less information. The task of deducing the binary formation mechanism from this data alone is akin to the problem of learning about star formation from the study of the IMF - in both cases, the problem is highly under-constrained, and a variety of prescriptions can reproduce functions of the correct form. Fortunately, the fact that one can in principle gather companion mass distributions for different primary masses goes quite a long way towards breaking this degeneracy. Although it would certainly be premature to make anything but the most preliminary judgments about the most likely binary formation mechanism, given present contradictions and confusions about the observational situation, one can at least set out the generic predictions of various classes of models. 
In this contribution, therefore, I shall summarise the predictions of various models and point out how current and future datasets may be used to discriminate between contending modes of formation. I shall also highlight a couple of other issues where I think observations are well placed to have an important impact on a theoretical understanding of binary star formation.

\section{The Models}

There are numerous ways to classify and describe binary star formation scenarios. Traditional classifications based on capture versus fragmentation are however becoming less useful, as simulations demonstrate both processes occurring on similar timescales during the first few dynamical times of a cloud's collapse. Indeed, since all star formation involves fragmentation, it is something of a truism to ascribe binary formation to fragmentation. Bearing in mind the need to define categories which have distinct observational predictions, I instead propose the following scheme and outline the predictied binary properties in each case:

\subsection{Capture}

I here restrict this term to describe the capture of two initially unbound objects, in the case that the capture event occurs after a time interval that is long compared with the internal collapse time of each object. For typical densities of molecular cloud cores, the latter is of order $10^{5}$ years, and so an immediate consequence of this scenario is that the binary components are non-coeval (at a level of non-coevality that is potentially detectable through comparison with pre-main sequence tracks). Another prediction of such delayed capture is that the binary should be devoid of a circumbinary disc: since the encounter occurs well after the initial protostellar collapse, the only distributed gas involved is that residing in low mass discs around each of the two stars, and simulations show that almost none of this is promoted into bound circumbinary orbit by the encounter (Hall, Clarke and Pringle 1996). At present the observational situation is unclear with respect to each of these issues (pre-main sequence tracks can be calibrated by the requirement that binary components are coeval (White et al. 1999) but, given the uncertainties in their construction do not at present provide independent verification of the coeval hypothesis (see contributions by Baraffe and Wuchterl; likewise, although massive circumbinary discs are rare, it is unclear whether low density material is commonly to be found in circumbinary orbit; see contribution by Jensen, this volume). However, such delayed capture models are probably ruled out as the primary binary star formation route by the simple fact that no proposed capture mechanism yields a high enough binary yield. The most promising mechanism is probably that of star-disc capture (Larson 1990), but even in the high density environment of the Orion Nebula Cluster this fails to produce a high enough binary fraction, mainly due to the fact that discs tend to be destroyed by high velocity non-capturing encounters before a slow capturing encounter has the chance to take place (Clarke and Pringle 1991a). Thus although it seems almost inevitable that such encounters will generate some binaries, particularly in high density cluster environments, we henceforth discount delayed capture as a major contributor to binary statistics. 


\section{2. $\quad N>2$ Fragmentation}

This category covers all scenarios in which a molecular cloud core splits into a small ensemble of fragments from which, following a sequence of dynamical interactions, a mixture of binaries, singles and higher order multiple systems emerge (e.g. Clarke and Pringle 1991b). Such behaviour is commonly found in numerical simulátions of cloud collapse (e.g. Burkert and Bodenheimer 1996), especially in the case of rather cold initial conditions. Possible observational evidence for an origin of stars in compact mini-clusters may be provided by the clustering of the youngest sources (see Reipurth, Mundy, this volume) as well as by the common existence of older hierarchical multiples (see Tokovinin, this volume).

Several processes may be at work in shaping the pairing characteristics of binaries formed in this way. In the absence of dissipative interactions, point mass gravitational interactions are on their own sufficient to guarantee that one binary forms from each mini-cluster, this binary containing the two most massive mini-cluster members (van Albada 1968). Obviously, the implied binary fraction depends on the membership number $(\mathrm{N})$ of the mini-clusters. Another obvious conseqeunce of this prescription is that the binary fraction is a steeply rising function of primary mass. Less obviously, a particular hallmark of such dynamical capture is that the secondary mass function is independent of primary mass, not only with respect to its shape but also its normalisation (McDonald and Clarke 1993). This would mean, for example, that the same fraction of $M$ stars and of $G$ stars would be expected to have brown dwarf companions in this scenario. [Note that this prediction is superficially at odds with the reported brown dwarf desert for $\mathrm{G}$ stars which may be contrasted with their relative abundance as secondaries to $\mathrm{M}$ dwarf primaries; see Basri, this volume. However, the quantity that is predicted to be invariant is the number of stars (of given mass) with companions in a given mass range as a fraction of all stars of that mass - i.e. including those that are binary secondaries. It is not clear whether the statistics are incompatible with this prediction when one takes account of those $\mathrm{M}$ dwarfs that are secondaries in higher mass binaries. This illustrates the difficulty of comparing even apparently simple model predictions with the data].

Alternatively, it is likely that in the gas rich conditions that prevail during the disintegration of mini-clusters, capturing encounters are likely to be mediated by dissipative interactions such as those involving the collisions of circumstellar discs. Crude parameterisations of such encounters in small $\mathrm{N}$ mini-clusters indicates that this modifies the pairing characteristics in several ways (McDonald and Clarke 1995): it increases the number of binary pairs per cluster (and hence allows a viable binary fraction from somewhat larger clusters) and softens the dependence of binary fraction on primary mass (since stars can now become binary primaries without having to be the most massive members of their minicluster). It also modifies the secondary mass distribution, so that in the case that dissipative interactions are sufficiently strong, the companion mass function becomes essentially proportional to the IMF for all primary masses.

We may summarise the observational predictions for $N>2$ fragmentation models as follows. The first three points apply also to $N=2$ fragmentation models (see below): 
a) Systems are predicted to be coeval to within $\sim 10^{5}$ years.

b) Circumbinary discs are not ruled out, as the encounters occur within a gas rich environment.

c) A high binary fraction is readily generated, unlike in the capture scenarios described above.

In addition, $N>2$ fragmentation models generically predict:

d) a binary fraction that increases with primary mass and

e) that the form of the secondary mass distribution should be invariant for different primary masses.

Differences between dissipationless and highly dissipative captures with regard to d) and e) are discussed above.

\section{3. $\quad N=2$ Fragmentation}

If binaries form primarily from the fragmentation of a cloud core into two pieces, then binary statistics are controlled by two quantities - the distribution of cloud core masses and the splitting function that describes the division of such cores into two. This scenario has been the subject of intense numerical investigation for more than twenty years (see Bodenheimer \& Burkert, this volume) and considerable progress has been made over that time, both in terms of achieving convergence between various codes and in terms of pursuing the binary collapse calculation through to completion (i.e. to the point where most of the mass is incorporated in one component or the other; see Bate, this volume). Nevertheless, even in the hypothetical case that one could use numerical simulations to credibly map given initial conditions onto a final binary product, one would still be no nearer to the goal of generating predictions for fragmentation models - essentially because any set of final parameters can be produced by suitable adjustment of the initial conditions. In the absence of independent knowledge of the initial conditions (particularly of the form of density perturbations at the onset of collapse) it would therefore seem that we can never test the hypothesis that binaries do indeed form in this way.

We can, however, make considerable progress by employing the additional assumption that the fragmentation process is scale free - i.e that the splitting function is independent of core mass. Provided that binaries form through fragmentation in the isothermal regime (which appears to be the case for all but possibly the closest binaries) then the splitting function depends only on a number of dimensionless numbers that parameterise the initial conditions, such as the ratio of thermal and rotational energy to the gravitational energy, and the amplitude and symmetry of the perturbations. If the distributions of these quantities do not vary systematically as a function of core mass then the resulting fragmentation is scale free.

Such a scenario (and indeed any $N=2$ fragmentation) gives rise to predictions a) to c) above. In addition, scale free fragmentation predicts:

d) the binary fraction is invariant with primary mass (when binary fraction is defined as the frequency of companions down to a fixed mass ratio limit) and

e) the companion mass ratio distribution is invariant for primaries of different masses. 
It should be noted that d) and e) are in strong contrast to the corresponding predictions for $N>2$ fragmentation detailed above.

Of course, the hypothesis that fragmentation is indeed scale free is itself open to question. One can certainly think of effects that are not blind to the total core mass, such as the importance of non-thermal support in the initial conditions and the effect of radiative feedback from the forming fragments. In the case of binaries that form from fragments in the adiabatic regime, as may be relevant to the closest binaries, the process is clearly not scale free, since the initial fragment mass is almost independent of core mass (Masunaga, Miyama \& Inutsuka 1998) and hence the ratio of subsequently accreted mass to initial mass is a strong function of core mass, with consequences for the mass ratio of the final binary (see Bate, this volume). Perhaps the investigation of mass dependent effects should be regarded as the next big challenge for those who model the fragmentation and collapse process.

\section{Resolving the Components}

Another area where observations can provide critical information about the formation and early evolution of young binaries is through high angular resolution studies of $\mathrm{T}$ Tauri binaries, which yield information about the circumstellar environment and accretion activity of the individual stellar components (see for example Duchene et al. 1999, Simon \& Prato 1995, Prato \& Simon 1997, Brandner \& Zinnecker 1997, Ghez, White \& Simon 1997). For most T Tauri binaries, such an enterprise requires the use of HST or ground based adaptive optics systems, and hence data has become available on this only rather recently. At the present time, the observational situation is rather confusing (see contributions by Patience, White and Hartigan, this volume). The majority of the data however seems to be consistent with a picture in which mixed pairs (i.e. those comprising a Classical T Tauri star - CTT - and Weak Line T Tauri star - WTT) are relatively rare, whilst in pairs that are doubly Classical (CTT-CTT) there is a tendency for the accretion signatures to be stronger on the binary primary.

These datasets will have an impact on a number of issues. Firstly, it might be thought that the relative strength of circumstellar material on the primary and secondary reflects the initial formation process. Simulations demonstrate a strong tendency for the primary to acquire the more massive disc (Bate 2000). It should be noted that although, as emphasised for example by Bate and Bonnell 1997 , the effect of infalling shells of high angular momentum is to preferentially deposit material around the secondary, more recent calculations, which follow the entire infall process, show that this effect is not sufficient to offset the advantage gained by the primary's disc early on in the infall - the net effect is that the primary's disc should always be more massive following the dynamical collapse phase of binary formation (see also Bate, this volume).

Alternatively, the mutual pairing properties of CTTs and WTTs may instead reflect the result of differential evolution in the discs around each component. Discs accrete onto their central stars on a timescale that is set by the viscous timescale at the disc's outer edge. In a binary star system, this outer edge is well defined by the gravitational potential of the binary system $(\mathrm{Pa}$ paloizou \& Pringle 1977): the primary's disc is always larger in spatial extent 
than the secondary's. As a result, the secondary's disc should always drain away more rapidly onto the star. Armitage, Clarke and Tout (1999) computed the photometric evolution of pairs of discs whose outer radii were set by the (invariant) mass ratio of the system and demonstrated that accretion signatures on the secondary should always fade away before those on the primary. This effect is more marked in close systems (because the overall clock for disc draining is faster in compact discs) and, in particular, for systems of extreme mass ratio. Clearly, these results are consistent with the observation that the primary in CTT-CTT systems should always have the stronger accretion signature, but is potentially inconsistent with the apparent scarcity of mixed CTT-WTT pairs the rapid draining of the secondary in these models should result in a number of mixed systems.

Two effects may be invoked to avoid the common production of mixed CTT-WTT pairs. One possibility is that the discs around both components are synchronously dispersed, before the system evolves into a mixed pair. It is at present unclear what process causes the rather rapid transition between the CTT and WTT state, as evidenced by the distribution of $\mathrm{T}$ Tauri stars in the infrared two colour plane (Simon and Prato 1995, Armitage et al. 1999). Whatever the nature of this process, the scarcity of systems that are WTT according to their near infrared characteristics (i.e. on small scales) but strong in the submillimetre (i.e. on scales of 10s of A.U.; Duvert et al. 2000) suggests that it operates rather synchronously over large regions within an individual disc: it is tempting to invoke a similar process that might simultaneously disperse two discs within a binary system.

Alternatively, as advocated by Prato and Simon (1997), the lack of mixed pairs might indicate replenishment of the circumstellar discs from some external reservoir during the T Tauri stage. The existence of CTTs in the closest binaries argues strongly that the discs in these systems are replenished, because the viscous timescales in these systems are so short; in these systems there appears to be plenty of material in circumbinary orbit to fuel such replenishment. In wider systems, it becomes harder to identify the source of replenishment, since massive circumbinary discs (such as that in GG Tau) appear to be the exception rather than the norm: SCUBA photometry should set firmer limits on the mass reservoirs in circumbinary discs. An attractive aspect of the idea of replenishment from circumbinary discs is that there is a tendency for accretion streams from such a disc to preferentially replenish the secondary's disc, in line with the requirement of avoiding the generation of mixed CTT-WTT pairs (note, however, that there is some evidence that this conclusion depends on the eccentricity of the binary orbit: see Artymowicz \& Lubow, this volume). Alternatively, Prato and Simon have argued for infall from an optically thin envelope. The origin of such infalling material is not obvious, however, since, at the age of the average $\mathrm{T}$ Tauri star (which is tens of dynamical times of its parent cloud core) one would not expect the residual dynamical infall to be large. Clearly, the issue of replenishment (which is potentially important, for example, for planet formation in binary environments) is far from settled at present. 


\section{Synthesizing the Field - Are the Wide Binaries a Problem?}

The collation of pre-main sequence binary statistics from different regions has opened up the possibility of using the binary properties of the field as a diagnostic of the mix of star forming environments that go to constitute the field. Such binary population synthesis arguments have been used to deduce that local field stars mainly originate from dense star forming regions (typified by the Orion Nebula Cluster, for example) where the binary fraction (in contrast to low density regions such as Taurus; Köhler \& Leinert 1998) appears to be compatible with the field (e.g. Petr et al. 1998). Such a notion is consistent with the idea that the bulk of star formation occurs in Giant Molecular Clouds, and that small scale regions, such as Taurus, are not major contributors to the Galactic star forming census.

These conclusions have however been based on binary surveys in Orion that only extend out to separations of $\sim 800$ A.U.. Wider binaries cannot be identified using positional data alone in crowded regions such as the ONC. The recent search for common proper motion pairs in the ONC by Scally, Clarke and McCaughrean (1999) however revealed a striking dearth of wider pairs (1000 5000 A.U. ) in that region. The data is consistent with no bound pairs in that separation range and is discrepant with the nominal binary fraction in that range for field stars reported by Duquennoy and Mayor (i.e. $7 \%$ ) at the $3 \sigma$ level.

Taken at face value, this result would indicate that no mixture of Orionlike and Taurus-like regions could be used to synthesise the field, since in both regions, the ratio of closer to wider pairs (i.e. those with separations $\sim 100$ A.U. to those with separations $>1000$ A.U.), is higher than in the field. One possible interpretation would be simply that the prototypical star forming region is different again from either Taurus or Orion (e.g. an OB association). On the other hand it should be stressed that the analysis of Scally et al. involved Monte Carlo simulations in which simulated data was viewed with errors applied according to the quoted error bars on Jones and Walker's proper motion data (Jones and Walker 1974). It would be therefore highly timely to repeat this analysis with higher precision proper motion data.

There is, however, a reason to suspect that the dearth of wide binaries in Orion is genuine, since Nbody simulations of the Orion Nebula Cluster by Kroupa, Petr and McCaughrean (1999) demonstrate that such wide binaries are likely to be dynamically disrupted, even if present at birth. In some ways this provides a reassuringly economical solution to the lack of wide binaries in Orion - one can invoke dynamical destruction and not concern oneself with differences in the initial binary formation process in different environments. On the other hand, this result does make the lack of wide pairs in Orion claimed by Scally et al. more credible, and so returns one to the problem of synthesising the field.

The final possibility is that we just don't know the field binary population very well at wide separations. The statistics compiled by Duquennoy and Mayor consisted of the results of their radial velocity surveys, augmented by wider pairs taken from the literature. Thus the wide pairs represent a less uniformly surveyed sample than the radial velocity pairs. It should be stressed that the $3 \sigma$ incompatibility claimed by Scally et al. represented the probability that the Orion data shared the same binary population at wide separations as nominal fraction for the field - i.e. it did not attempt to place error bars on the field 
data because it was not clear how that should be done. If the synthesis of the binary properties of field stars is an important element in determining the types of environments in which stars mainly form, then it is clearly vital that our knowldege of the field and its incompleteness be as reliable as possible. The urgent need now would seem to be to improve the field binary statistics at large separations, since it is these binaries which, being most prone to dynamical disruption, are likely to be the most sensitive probes of star forming environment.

\section{References}

Armitage, P. J., Clarke, C. J., \& Tout, C. A. 1999, MNRAS, 304, 425

Bate, M. R. 2000, MNRAS, in press (astro-ph/0002143)

Bate, M. R., \& Bonnell, I. A. 1997, MNRAS, 285, 33

Brandner, W., \& Zinnecker, H. 1997, A\&A, 321, 220

Burkert, A., \& Bodenheimer, P. 1996, MNRAS, 280, 1190

Clarke, C. J. 1996, MNRAS, 282, 353

Clarke, C. J., \& Pringle, J. E. 1991a, MNRAS, 249, 584

Clarke, C. J., \& Pringle, J. E. 1991b, MNRAS, 249, 588

Duchene, G., Monin, J-L, Bouvier, J., \& Menard, F. 1999, A\&A, 351, 954

Duquennoy, A., \& Mayor, M. 1991, A\&A, 243, 485

Duvert, G., Guilloteau, S., Menard, F., Simon, M., \& Dutrey, A. 2000, A\&A, 355,165

Ghez, A. M., White, R. J., \& Simon, M. J. 1997, ApJ, 490, 353

Hall, S. M., Clarke, C. J., \& Pringle, J. E. 1996, MNRAS, 278, 303

Jones, B. F., \& Walker, M. F. 1988, AJ, 95, 1755

Köhler, R., \& Leinert, Ch. 1998, A\&A, 331, 977

Kroupa, P., Petr, M. G., \& McCaughrean, M. J. 1999, New Astronomy, 4, 495

Larson, R. B. 1990, Formation of star clusters in Physical Processes in Fragmentation and Star Formation ed. R. Capuzzo-Dolcetta, C. Chiosi \& A. DiFazio, Kluwer, Dordrecht, 389

Masunaga, H., Miyama, S., \& Inutsuka, S. 1998, ApJ, 495, 346

McDonald, J. M., \& Clarke, C. J. 1993, MNRAS, 262, 800

McDonald, J. M., \& Clarke, C. J. 1995, MNRAS, 275, 671

Papaloizou, J. C. B., \& Pringle, J. E. 1977, MNRAS, 181, 441

Petr, M. G., du Foresto, V. C., Beckwith, S. V. W., Richichi, A., McCaughrean, M. J. 1998, ApJ, 500, 825

Prato, L., \& Simon, M. 1997, ApJ, 474, 455

Scally, A., Clarke, C. J., \& McCaughrean, M. J. 1999, MNRAS, 306, 253

Simon, M., \& Prato, L. 1995, ApJ, 450, 475

van Albada, T. S. 1968, Bull. Astron. Inst. Netherlands, 20, 57

White, R. J., Ghez, A. M., Reid, I. N., \& Schultz, G. 1999, ApJ, 520, 811 


\section{Photographic Intermezzo}
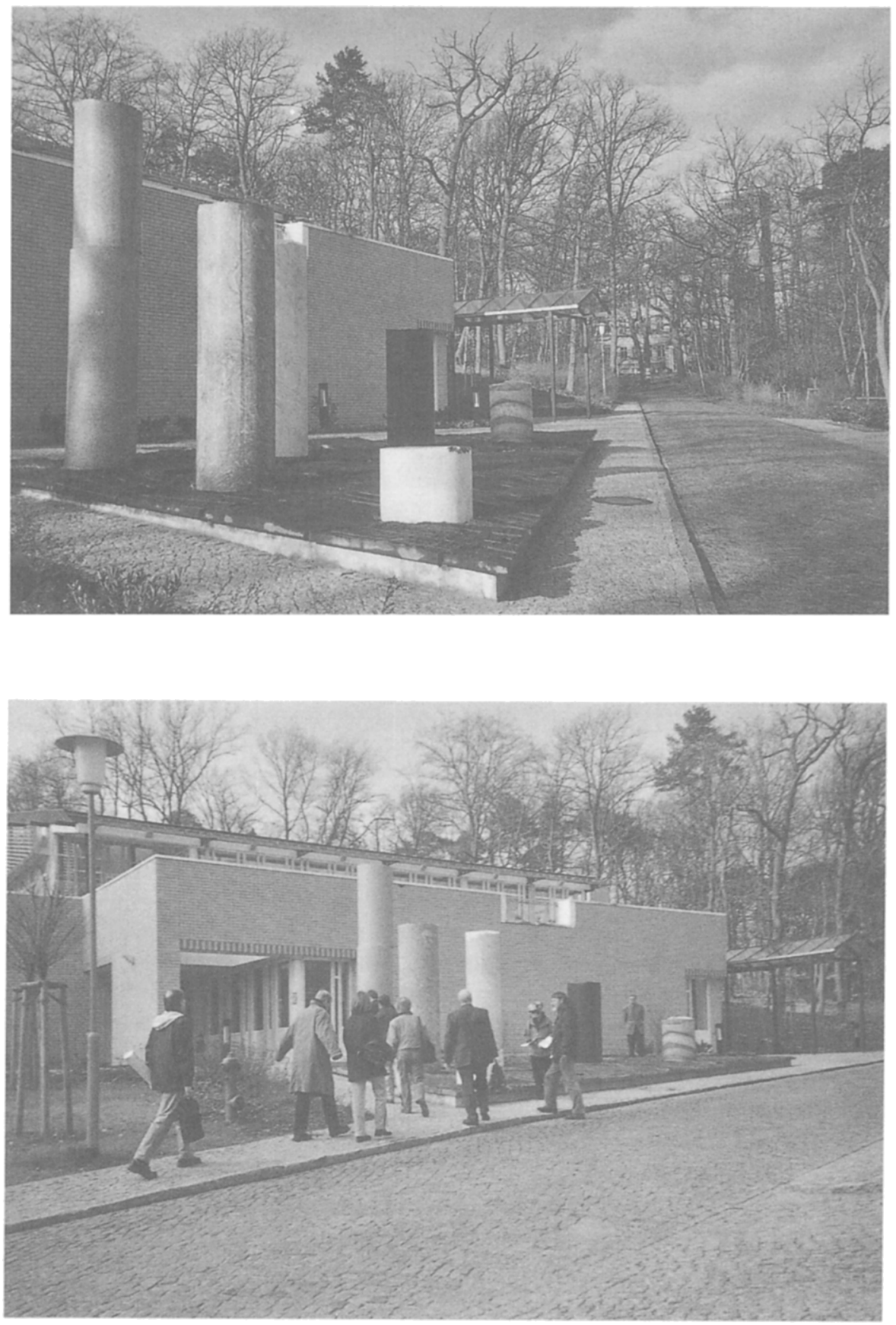

Venue at Telegrafenberg 

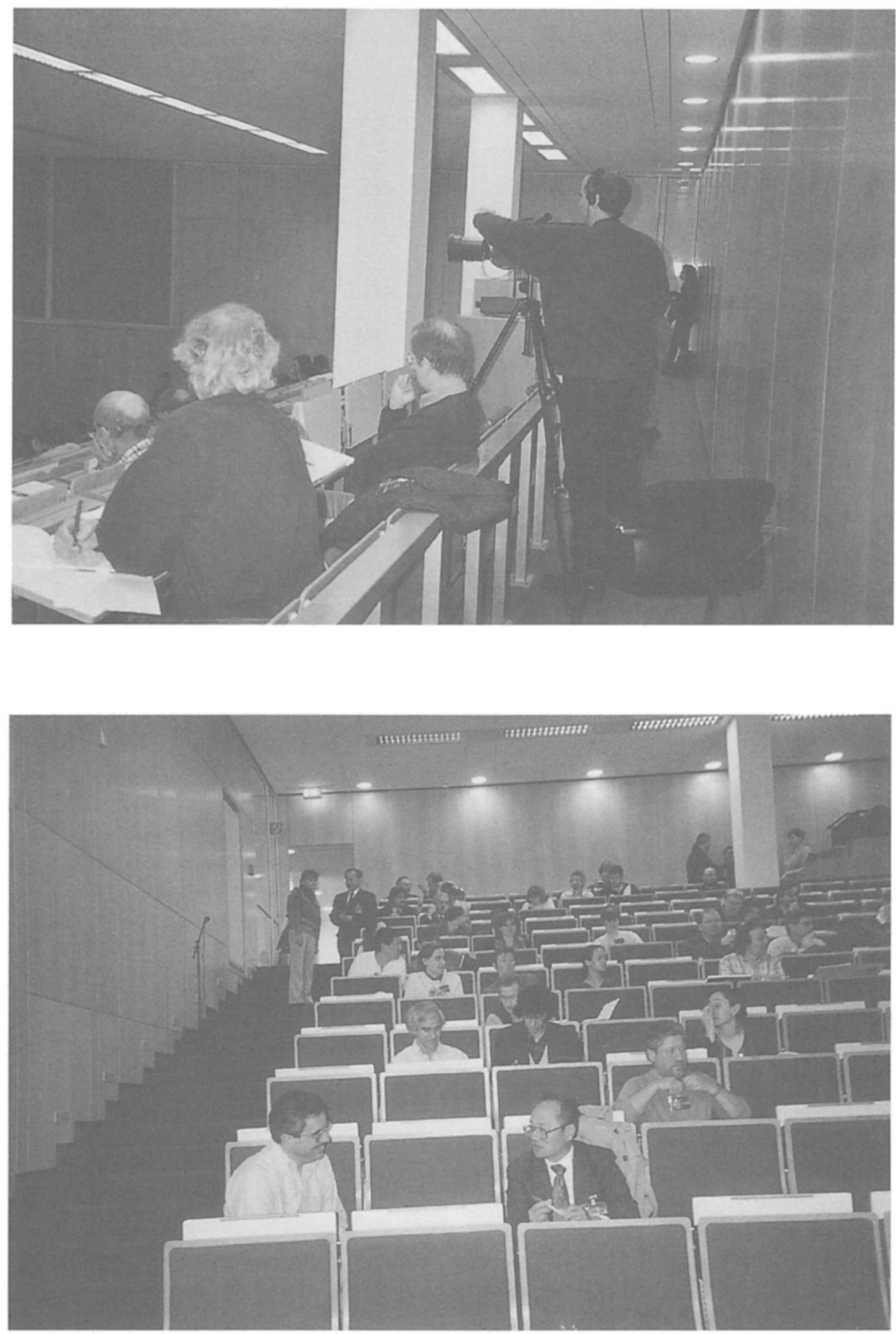

\section{Lecture Hall}



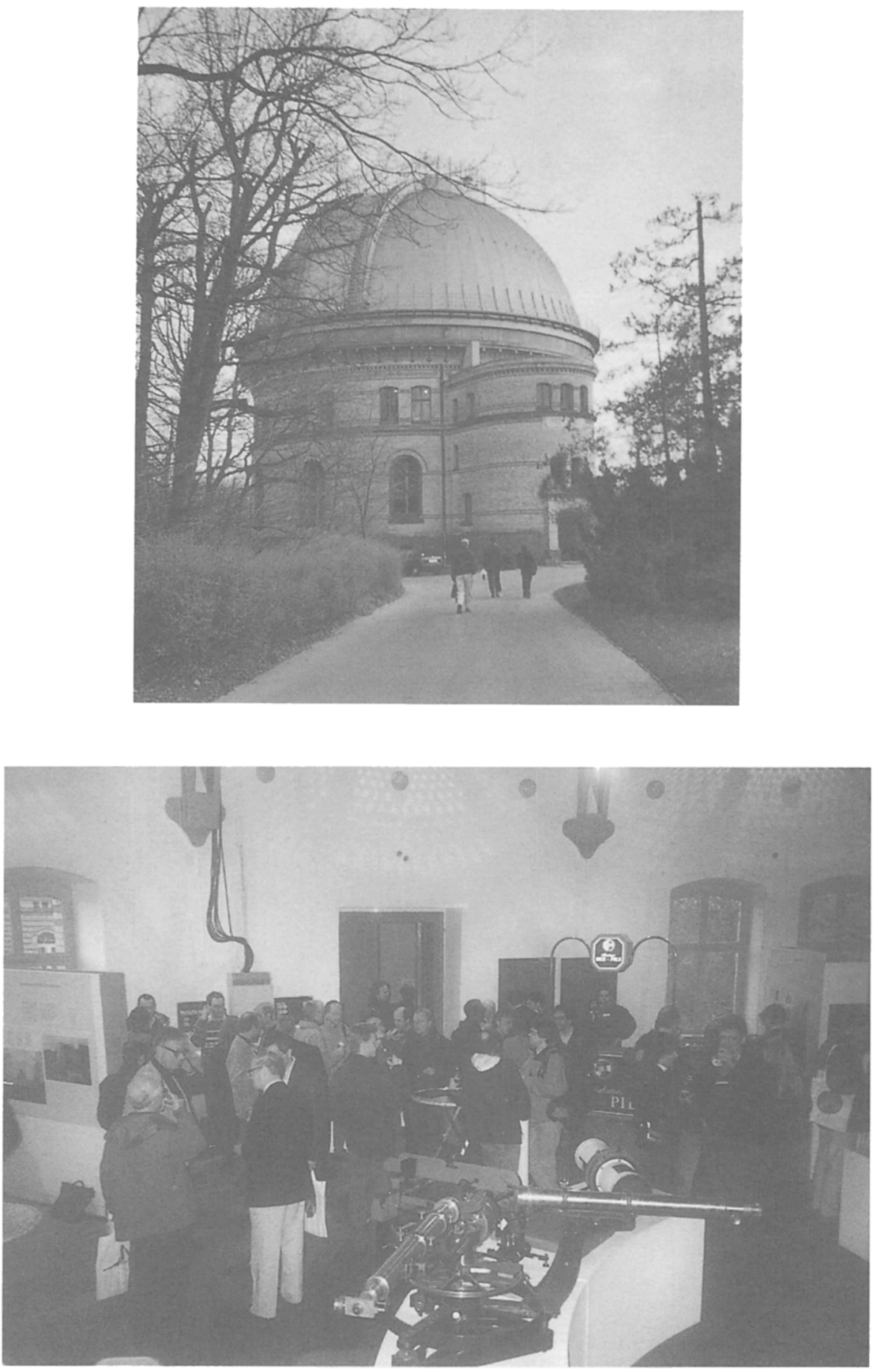

Welcome Party in the Refractor Dome 

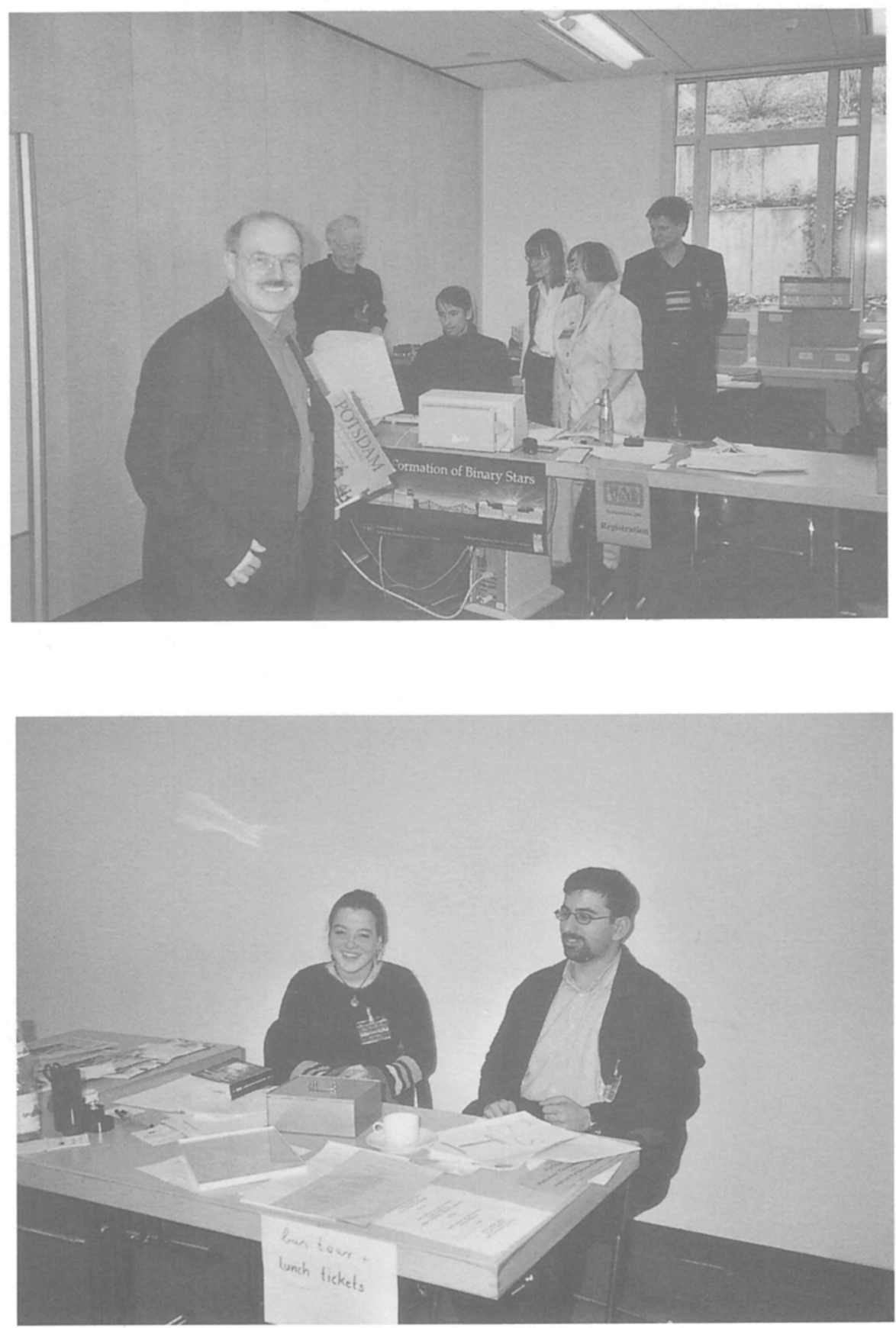

Conference Office 

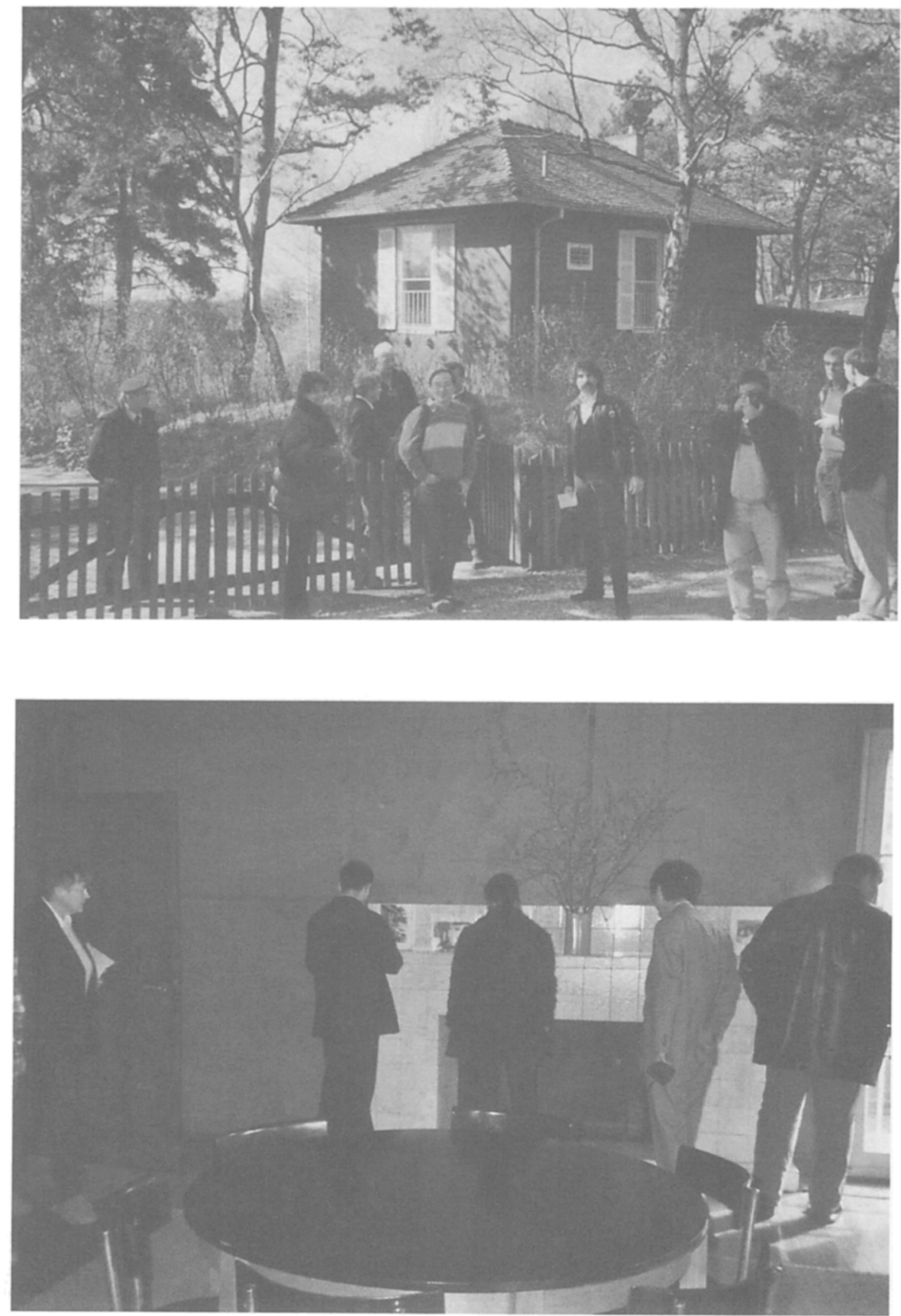

Excursion: Einstein's Summer Cottage in Caputh 

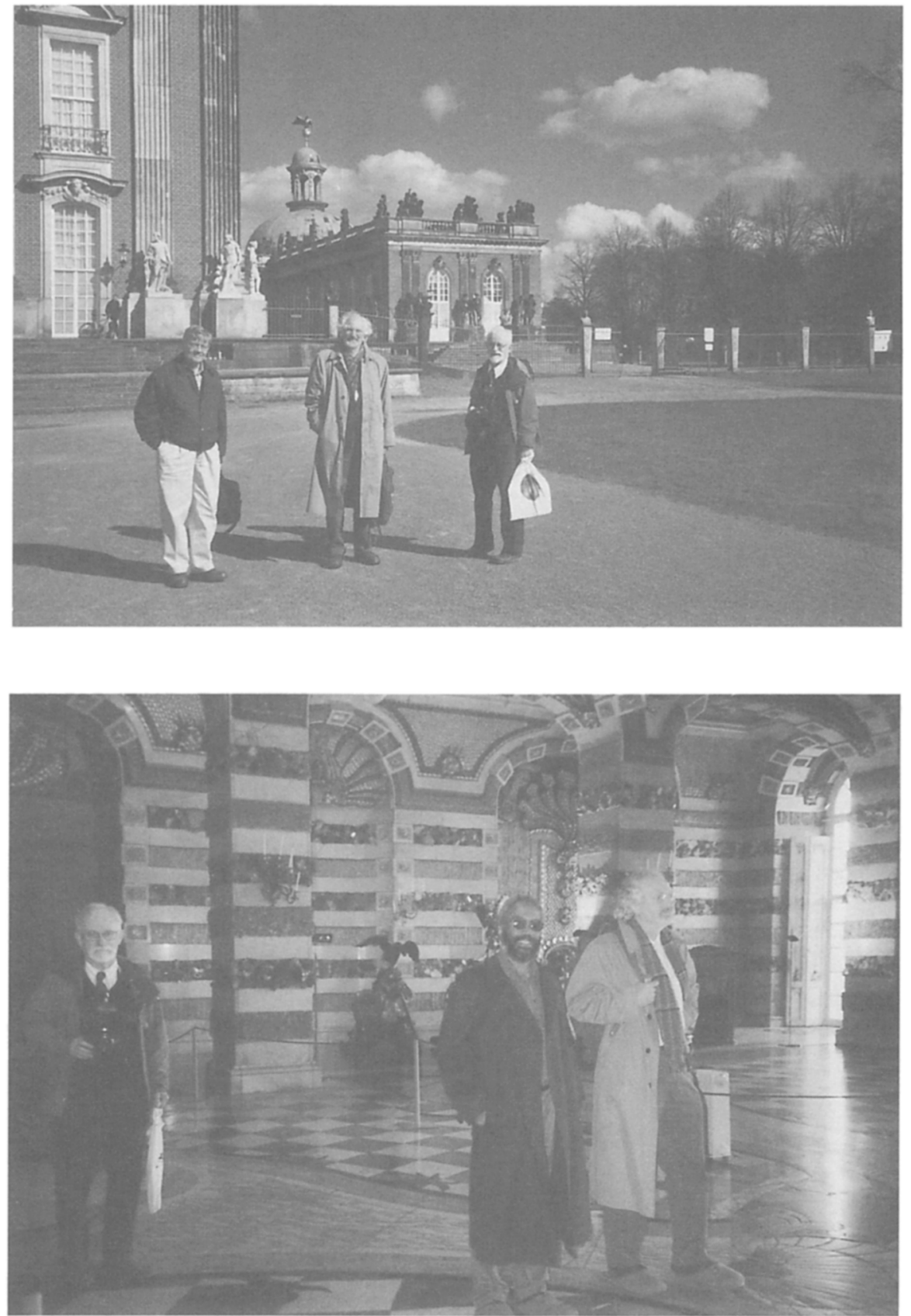

Excursion: Neues Palais / Potsdam, Sanssouci 

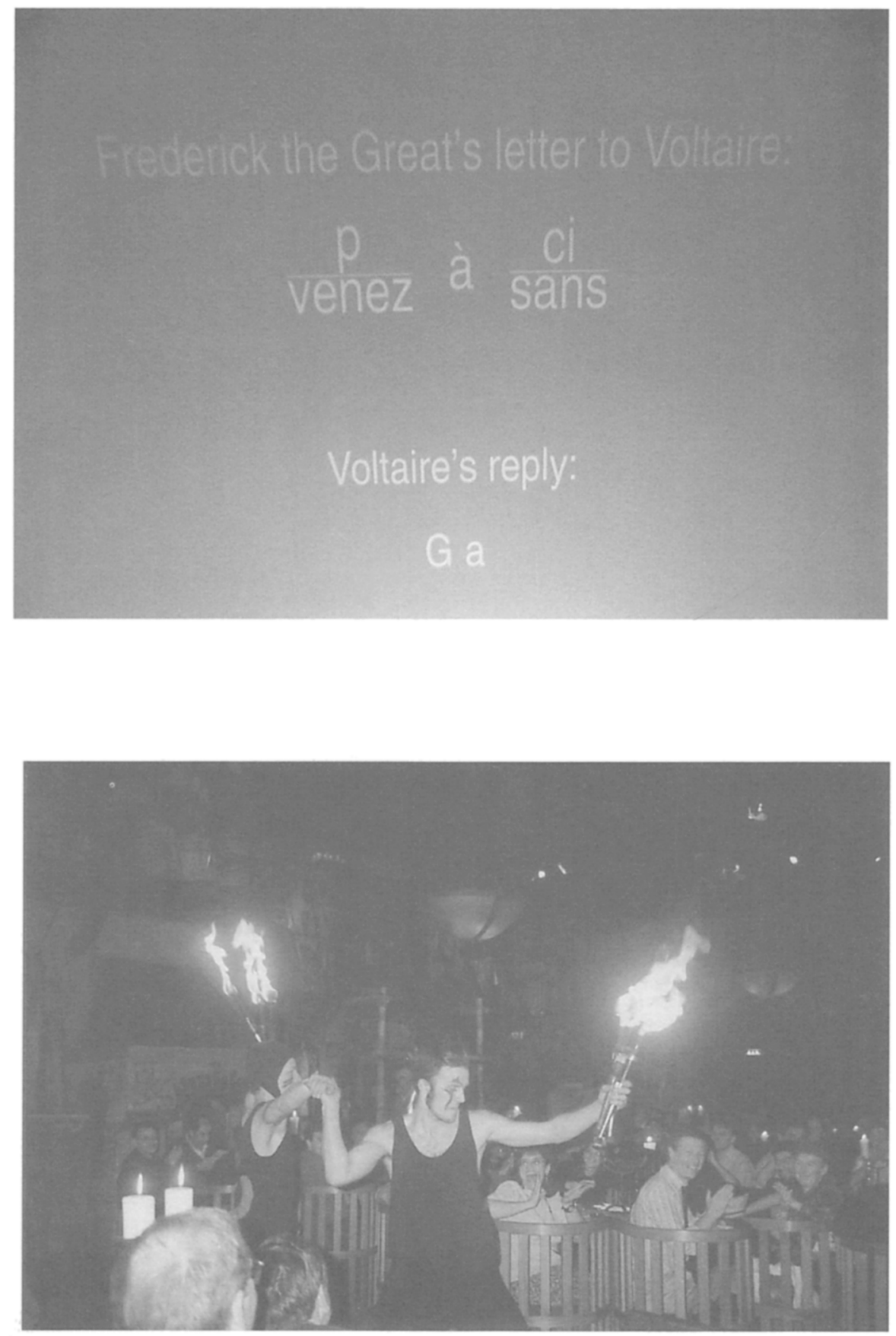

Conference Dinner and Entertainment 